\title{
ANALYSIS OF NOISE AND INTERFERENCE IN COMPANDING SIGNAL PROCESSORS
}

\author{
L. Tóth ${ }^{1}$, Y. Tsividis ${ }^{2}$, N. Krishnapura ${ }^{2}$ \\ ${ }^{1}$ CSRL, Lucent Technologies, Bell Laboratories, Murray Hill, NJ 07974, USA \\ ${ }^{2}$ Department of Electrical Engineering, Columbia University, New York, NY, 10027, USA
}

\begin{abstract}
The analysis of the effects of noise and interference in companding signal processors are discussed. Important differences from the analysis and effects encountered in conventional signal processors are pointed out. Finally, the theoretical calculations are successfully compared to experimental results.
\end{abstract}

\section{INTRODUCTION}

Log-domain and other types of companding signal processing has been proposed as a way of maintaining adequate dynamic range in integrated circuits with low power supply voltages $[1,2,3,4]$. Although such circuits perform linear operations on the input signal, they are nonlinear from internal points to the output. This fact leads to unusual behavior with respect to noise and interference [5]. In this paper, an investigation of this behavior is undertaken. The inadequacy of classical analysis techniques, commonly applied to conventional signal processors, are pointed out, and methods for the analysis of noise and interference in companding signal processors are discussed.

Throughout this paper, a first order system is used as an example (Fig. 1). This is done both in order not to allow complexity to obscure the results, and because integrators are the dominant building blocks in high-order filter structures. The application of the discussed techniques to higher order structures is, in principle, straightforward, albeit lengthy.

The transfer function of the system in Fig. 1 is

$$
H(s)=\frac{k}{s+a}
$$

and in the time domain, the system can be described by

$$
\begin{aligned}
\dot{x} & =-a x+k u \\
y & =x
\end{aligned}
$$

where $x$ is the state variable, $\dot{x}$ is its time derivative and $k$ $\& a$ are known constants. We now consider a system with the same input-output behavior, but with a state variable $v$, which is related to $x$ by $[3,4]$ :

$$
x=f(v)
$$

where $f(v)$ is a monotonic function with continuous nonzero derivative for all $v$. Using (4) in (1) gives:

$$
\dot{v}=-a \frac{f(v)}{f^{\prime}(v)}+\frac{k}{f^{\prime}(v)} u
$$

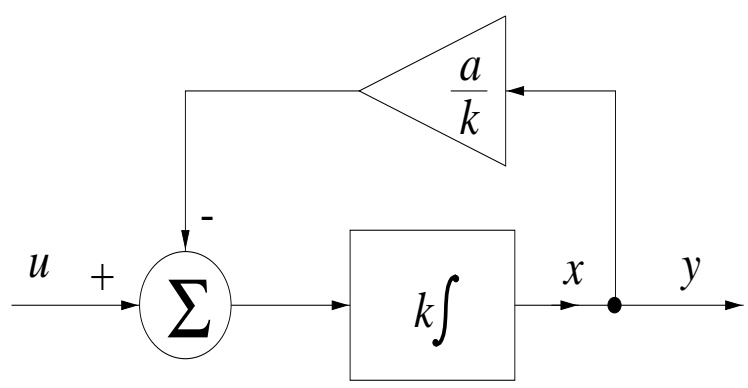

Figure 1. Linear first order system

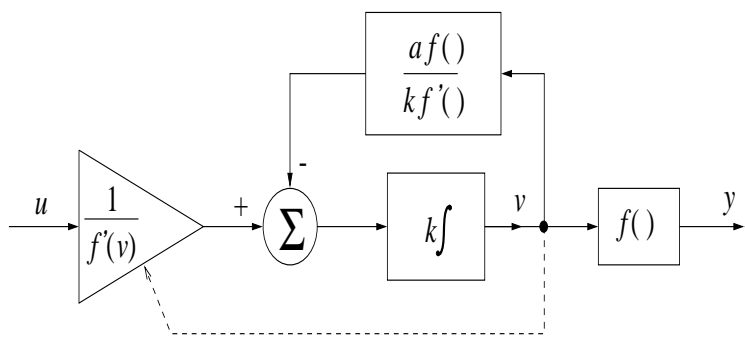

Figure 2. Input-output linear first order system

$$
y=f(v)
$$

where $f^{\prime}(v)$ is the derivative of $f(v)$ with respect to $v$ [4]. The behavior between $u$ and $y$ is linear and is still described by (2) and (3) $[4,5]$. An implementation of (5) and (6) is shown in Fig. 2.

We now consider the effects of small additive interference, referred to as "noise", on this system. It is obvious that noise at the output is simply added to $y$, and that noise at the input is processed along with the signal by the transfer function in (1). However, noise at internal points undergoes nonlinear signal processing. This is demonstrated by considering two representative cases in the following section.

\section{TIME RESPONSE TO SMALL INTERFERENCE AND NOISE AT INTERNAL POINTS}

Consider a noise $n_{1}$ added at the input of the $f()$ block in Fig. 2. This noise source does not affect anything to the left of it, so $v$ is not influenced by it. The output is $y_{1}=f\left(v+n_{1}\right)$. Assuming that $n_{1}$ is sufficiently small, we can use the approximation $y_{1}=f(v)+f^{\prime}(v) n_{1}$. The amount by which this differs from the output of the noiseless system $y$ in (6), is the output noise, $n_{o 1}$, given by 


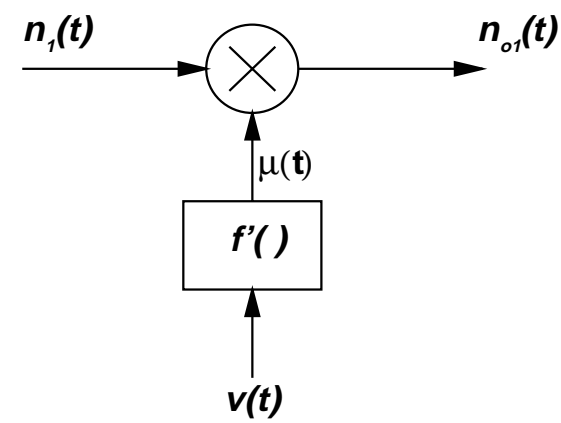

Figure 3. Noise (small signal) equivalent system representing $(7)$

$$
n_{o 1}(t)=\mu(t) n_{1}(t)
$$

where:

$$
\mu(t)=f^{\prime}(v(t))
$$

Note that $n_{o 1}$ results from memoryless processing of $n_{1}$. This processing can be represented as in Fig. 3 .

Consider now an additive noise $n_{2}$ at the summer of Fig. 2. This can represent external interference, or the output noise of the left-hand block, or the output noise of the upper block, or the equivalent input referred noise of the integrator. Now the state variable $v_{2}(t)$ is affected, and will be different from $v(t)$ in (5) and (6). Writing the state equations gives:

$$
\begin{aligned}
\dot{v_{2}} & =-a \frac{f\left(v_{2}\right)}{f^{\prime}\left(v_{2}\right)}+\frac{k}{f^{\prime}\left(v_{2}\right)} u+k n_{2} \\
y_{2} & =f\left(v_{2}\right)
\end{aligned}
$$

Defining the output noise (relative to the noiseless system in Fig. 2) as $n_{o 2}=y_{2}-y$, combining (5) and (6) with (9) and (10) and using the same Taylorapproximation as in obtaining (7), we get:

$$
\frac{d}{d t} n_{o 2}(t)=-a n_{o 2}(t)+k \mu(t) n_{2}(t)
$$

where, $\mu(t)$ has been defined in (8). Thus $n_{02}$ is a result of processing $n_{2}$ by a system with memory. The relation in (11) can be represented by the equivalent system shown in Fig. 4.

In a sense, the noise $n_{2}$ is "amplified" by the signal dependent gain factor $\mu(t)=f^{\prime}(v(t))$ and filtered by $H(s)$.

It can be seen from Fig. 3 and Fig. 4 that for small $n_{1}$ and $n_{2}$ the noise inputs are processed by linear time varying (LTV) systems, since $v(t)$ is known from the noiseless system equations in (5) and (6). If $\mu(t)$ is periodic with a period $T$, the LTV system becomes linear periodically time varying (LPTV).

\section{RESPONSE TO STATIONARY WHITE NOISE IN THE PRESENCE OF A PERIODIC INPUT}

If $n_{1}$ or $n_{2}$ in Fig. 3 or Fig. 4 is random noise, the evaluation of the output noise can in general be very complicated. We have found explicit expressions under the following two assumptions:

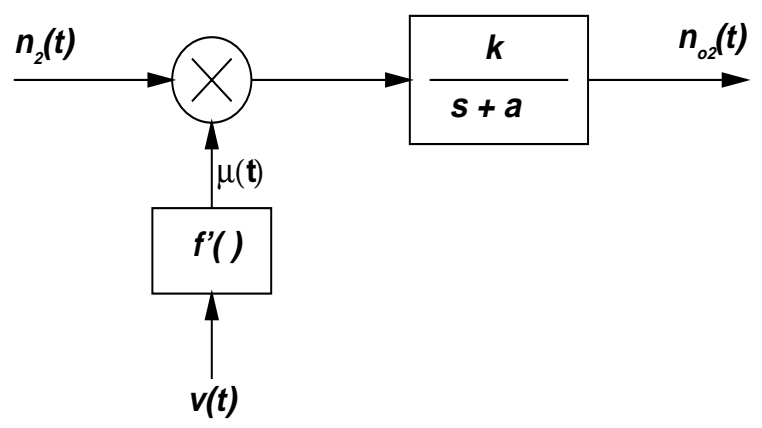

Figure 4. Noise (small signal) equivalent system representing (11)

1. The noise is wide-sense stationary and white.

2. The input $u(t)$ is periodic, and the corresponding noiseless system (Fig. 2) has reached steady state.

In such cases, we can evaluate the output noise power spectral density (PSD) as [7]:

$$
S_{o}(\omega)=S_{n} \frac{1}{T} \int_{0}^{T}|H(\omega, \tau)|^{2} d \tau
$$

where, $S_{n}$ is the PSD of the noise excitation, $T$ is the period of the input (hence the period of $v(t)$ ), and $H(\omega, \tau)$ is the Fourier transform of the response of the corresponding small-signal LPTV system (see Fig. 3, Fig. 4) to a shifted delta function $\delta(t-\tau)$.

For the systems in Fig. 3 and Fig. 4 after replacing $n_{1}$ and $n_{2}$ by $\delta(t-\tau)$, the corresponding system responses are $h_{1}(t, \tau)=\mu(\tau) \delta(t-\tau)$ and $h_{2}(t, \tau)=$ $\mu(\tau) h(t-\tau)$, where $h(t)$ is the inverse Laplace transform of $H(s)$. Thus, the corresponding Fourier transforms are $H_{1}(\omega, \tau)=e^{-j \omega \tau} \mu(\tau)$ and $H_{2}(\omega, \tau)=e^{-j \omega \tau} \mu(\tau) H(j \omega)$. Substituting these into (12) for $H(\omega, \tau)$ and using $H(j \omega)$ in (1), the noise PSDs of $n_{o 1}$ and $n_{o 2}$ become

$$
S_{o 1}(\omega)=\eta S_{1} \quad, \quad S_{o 2}(\omega)=\eta \frac{k^{2}}{a^{2}+\omega^{2}} S_{2}
$$

respectively, where:

$$
\eta=\frac{1}{T} \int_{0}^{T}|\mu(\tau)|^{2} d \tau
$$

It can be seen from (8), (13) and (14) that the amount of output noise depends on the size and the shape of the signal. If more than one signal is present at the input, the combined signal determines the output noise, through $v(t)$ in (8) and (14). The possibility thus exists that the output noise caused by the presence of a large signal can "drown out" a small signal at the output.

\section{EXAMPLE AND EXPERIMENTAL RESULTS}

Consider the log-domain circuit in Fig. 5 [3, 8]. The quantity $i_{n 2}$ represents a noise current which, for example, can originate in the devices that develop $I$, or can represent external interference. Assume first that $i_{n 2}=0$; an analysis 


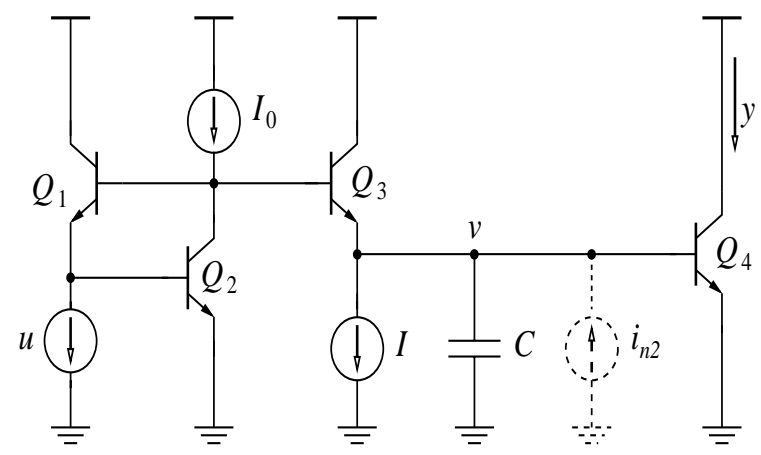

Figure 5. First order Class-A log-domain filter

$[3,8,9]$ (neglecting all the base currents) gives equations of the form of (5) and (6), with $f(v)=I_{s} e^{\frac{v}{V_{T}}}, k=\frac{I_{0}}{C V_{T}}$ and $a=\frac{I}{C V_{T}}$, where $I_{s}$ is the reverse saturation current of the transistors (assumed the same for all transistors) and $V_{T}$ is the "thermal voltage" (26 $\mathrm{mV}$ at room temperature). If $i_{n 2} \neq 0$, these equations are in the form of (9), (10) with $n_{2}=\frac{V_{T}}{I_{0}} i_{n 2}$. From these relationships we have:

$$
H(j \omega)=\frac{I_{0} / I}{1+j \omega\left(C V_{T} / I\right)}
$$

Consider an input of the form:

$$
u(t)=I_{c u}+I_{p u} \cos (\omega t)
$$

Using (15) we obtain for the noiseless case that $y(t)=$ $I_{c y}+I_{p y} \cos (\omega t+\phi)$ where:

$I_{c y}=\frac{I_{0}}{I} I_{c u}, \quad I_{p y}=\frac{I_{0} / I}{\sqrt{1+\omega^{2}}}, \phi=-\tan ^{-1}\left(\omega\left(C V_{T} / I\right)\right)$

From (8), $y=f(v)=I_{s} e^{\frac{v}{V_{T}}}$ and (17) we have $\mu(t)=$ $\frac{I_{c y}}{V_{T}}+\frac{I_{p y}}{V_{T}} \cos (\omega t+\phi)$. This can be used in (14) to calculate $\eta$. The result is:

$$
\eta=\frac{1}{V_{T}^{2}}\left(I_{c y}^{2}+\frac{1}{2} I_{p y}^{2}\right)
$$

A related result for this special case has been found in $[10]$.

Measurements were performed on the circuits of Fig. 5 using $I_{0}=20 \mu A, I_{1}=20 \mu A$. These values result in $k=a=2 \pi \times 12.3 \mathrm{krad} / \mathrm{s}$. The noise injected was deliberately made large enough in order to dominate all other noise in the circuit and enable a comparison to the analytical results. White noise with a PSD of $0.35 \mathrm{nA} / \sqrt{\mathrm{Hz}}$ was injected as shown in Fig. 5. The input to the filter was $20 \mu \mathrm{A}+5 \mu \mathrm{A} \cos (2 \pi \times 6 \mathrm{kHz} \times t)$. Using (13), (17) and (18), the output noise PSD was calculated. The calculated curve and the measured points are shown in Fig. 6.

Fig. 7 shows a first order class-AB log-domain filter[3], where $i_{n 2}$ is a noise current. A straightforward analysis results in the following

$$
\begin{aligned}
\frac{d}{d t} y_{a 2} & =k u_{a}-a y_{a}-\frac{1}{C V_{T}} y_{a} y_{b}+\frac{1}{C V_{T}} y_{a} i_{n 2} \\
\frac{d}{d t} y_{b 2} & =k u_{b}-a y_{b}-\frac{1}{C V_{T}} y_{a} y_{b}
\end{aligned}
$$

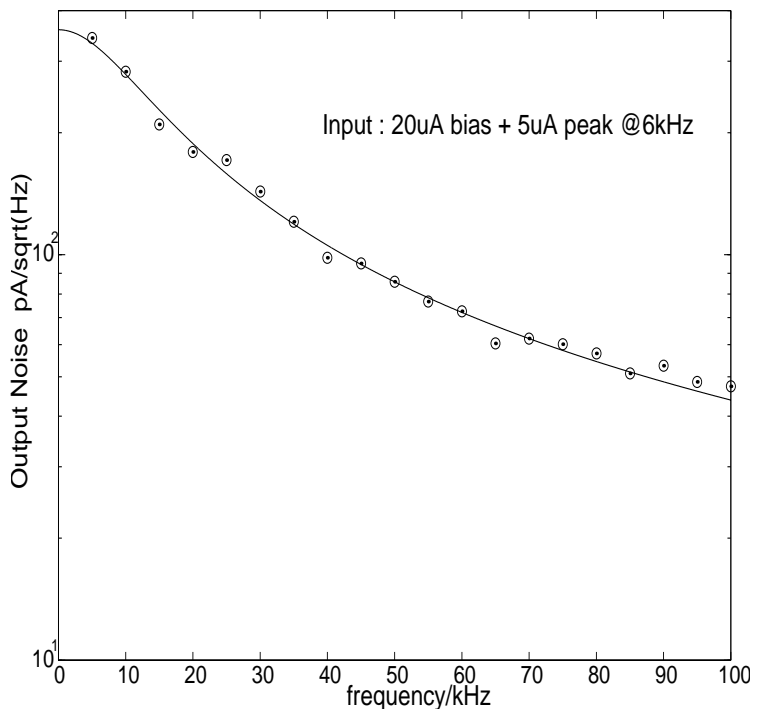

Figure 6. Output noise current PSD for the ClassA log-domain circuit. Line: calculated, Circles: measured values.

As is shown in [3], this is a linear first order filter, with $u(t)=u_{a}(t)-u_{b}(t)$ as the input and $y_{2}(t)=y_{a}(t)-$ $y_{b}(t)$ as the output. By making approximations similar to that used above, we get

$$
\frac{d}{d t} y_{2}=-a y_{2}+k u+k f^{\prime}\left(v_{a}\right) n_{2}
$$

It can be shown that, for low frequencies (in comparison with the cutoff frequency of the filter), the two halves of the circuit operate on alternating half cycles of the input current $u(t)$. With this approximation, the integral in (14) becomes proportional to the integral of $y_{a}^{2}(t) / V_{T}^{2}$ where $y_{a}$ is a half sine wave. Once $\eta$ is obtained, the output noise PSD can be computed in a manner described in the earlier section.

Fig. 8 shows the calculated and measured noise PSD for a class-AB first order log-domain filter, for two different values of peak input currents and $i_{n 2}=$ $0.35 \mathrm{nA} / \sqrt{\mathrm{Hz}} . I_{0}$ and $I_{1}$ are the same as in the class-A circuit, and hence, the transfer function realized is the same as before. It is clearly seen that the output noise increases with the magnitude of the output signal. This fact is not readily apparent in a class-A system because the signal is constrained to be within the limits of the bias current. As can be seen from (18), $\eta$ can vary only between 1.0 and 1.5 (a change of $1.8 \mathrm{~dB}$ ), as $I_{p y}$ varies between 0 and $I_{c y}$.

\section{CONCLUSION}

Conventional techniques, used for the analysis of noise in linear circuits, cannot be used for companding signal processors. This is because the latter, although externally linear, exhibit nonlinear behavior between internal points and the output. Appropriate techniques for the analysis of noise and small interference in companding signal processors have been discussed. Explicit relations were given 


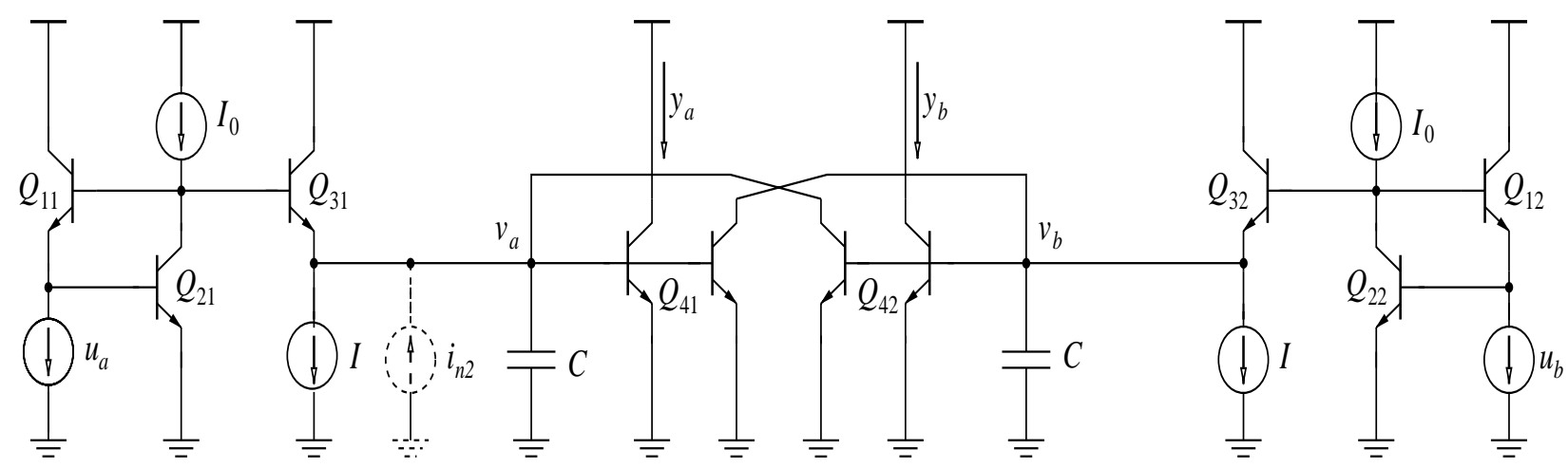

Figure 7. Class-AB first order $\log -$ domain filter

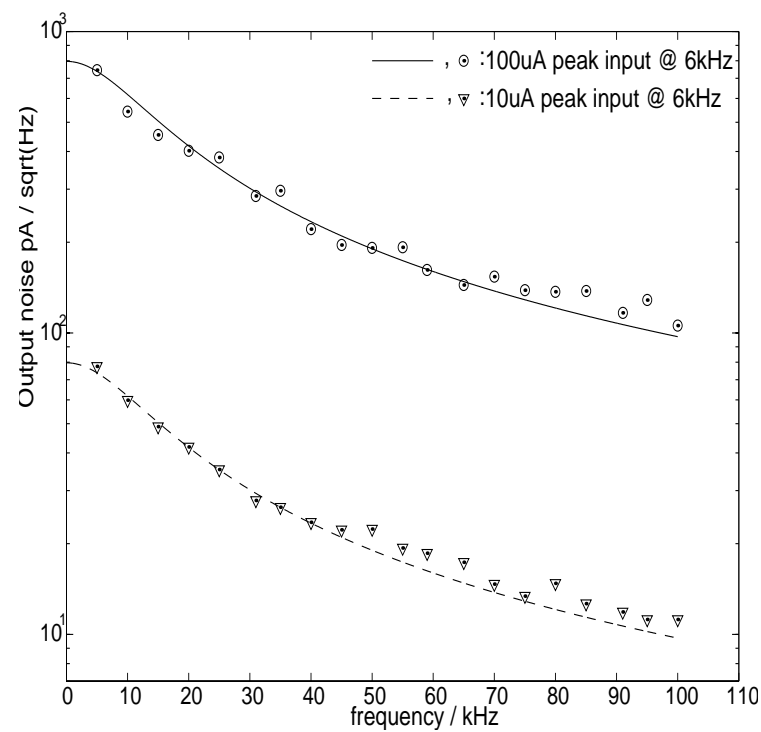

Figure 8. Output noise current PSD for the ClassAB log-domain circuit. Line: calculated, Circles: measured values.

for the case of stationary white noise with a periodic input. It is found that, in general, the output noise of such systems depends on the amplitude and the shape of the output signal. The predictions were verified experimentally using a first order log-domain filter as an example.

\section{REFERENCES}

[1] R. W. Adams, "Filtering in the Log Domain", Preprint 1470, presented at $63^{\text {rd }}$ Audio Engineering Society Conference, NY, May, 1979.

[2] Y. P. Tsividis, V. Gopinathan, and L. Tóth, "Companding in Signal Processing", Electronics Letters, vol. 26, pp. 1331-1332, Aug. 1990.

[3] E. Seevinck, "Companding Current-Mode Integrator: A New Circuit Principle for Continuous-Time Monolithic Filters", Electronics Letters, vol. 26, pp. 2046-2047, Nov. 1990.
[4] D. R. Frey, "A General Class of Current Mode Filters", in Proc. IEEE 1993 ISCAS, pp. 1435-1438, Chicago, IL, May 1993.

[5] Y. Tsividis, "Externally Linear, Time-Invariant Systems and Their Application to Companding Signal Processors", IEEE TCAS-II, vol. 44, no. 2, pp. 6585, Feb. 1997.

[6] L. A. Zadeh and C. A. Desoer, Linear System Theory: The State Space Approach, McGraw-Hill, 1963.

[7] S. O. Rice, "Response of Periodically Varying Systems to Shot Noise-Application to Switched RC Circuits", Bell System Technical Journal, vol. 49, no. 9, pp. 2221-2247, Nov. 1970.

[8] F. Yang, C. Enz, and G, van Ruymbeke, "Design of Low-Power and Low-Voltage Log-Domain Filters", in Proc. 1996 IEEE ISCAS, pp. 117-120, Atlanta, GA, May 1996.

[9] D. R. Frey, "Exponential State Space Filters: A Generic Current Mode Design Strategy", IEEE TCAS-I vol. 43, pp. 34-42, Jan. 1996.

[10] M. Punzenberger and C. Enz, "A $1.2 \mathrm{~V}$ BiCMOS Class-AB Log-Domain Filter", Digest 1997 ISSCC, pp. 56-57, San Francisco, Feb. 1997, pp. 56-57, and slide supplement, p. 34 